\title{
Simultaneous air/fuel-phase PIV measurements in a dense fuel spray
}

\author{
K.D. Driscoll, V. Sick, C. Gray
}

\begin{abstract}
A new diagnostic has been developed that is capable of obtaining simultaneous two-phase velocity measurements in a gasoline direct-injection fuel spray. This technique utilizes a two-laser (double-pulse) twocamera (double-frame) setup to simultaneously image the injected fuel and entrained air to determine the $2 \mathrm{D}$ velocity vector fields of both phases using cross-correlation particle image velocimetry (PIV). The air phase is visualized through fluorescence from seeding particles introduced into the static measurement volume while Mie scattering signals are collected from the fuel droplets. The combination of different laser wavelengths and a spectral signal shift for the air phase allows spectral separation of the signals. Independent timing of the laser pulses permits optimized adaptation of the velocity dynamic range for the two phases to account for the large difference in velocities between air and fuel droplets.
\end{abstract}

\section{1}

\section{Introduction}

The breakup of liquid fuel into droplets and the spatial distribution of these droplets depend on several factors, including the momentum exchange between the liquid phase and the surrounding gas phase. In modern fuel injectors the fuel is pressurized to several $\mathrm{MPa}$, which leads to relative velocities between fuel and the surrounding gas on the order of $100 \mathrm{~m} / \mathrm{s}$. The rapidly propagating fuel induces a motion in the surrounding gas, adding substantial levels of turbulence to the system (Miles et al. 2001), which in turn can lead to secondary effects on the developing spray itself. An example of this effect is the well-known roll-up vortex in pressure-swirl

Received: 29 July 2002 / Accepted: 31 March 2003

Published online: 18 June 2003

(C) Springer-Verlag 2003

K.D. Driscoll, V. Sick ( $\square)$

2023 W. E. Lay Automotive Laboratory,

University of Michigan, 1231 Beal Ave.,

Ann Arbor, MI 48109-2133, USA

E-mail: vsick@umich.edu

C. Gray

LaVision Inc., 301 W. Michigan Ave., Suite 403,

Ypsilanti, MI 48197, USA

This work was supported by General Motors Corporation within the General Motors Collaborative Research Laboratory at The University of Michigan. KDD's work benefited from a visit to the University of Heidelberg, funded via a grant from NSF INT9815290 injectors (Zhao et al. 2002). A faithful simulation of sprays will need to predict the surrounding flow field and its impact on the spray evolution. Thus, experimental data for comparison with advanced models need to characterize not only the velocity of the fuel droplets but also (ideally simultaneously) the velocities in the gas phase. Particle image velocimetry (PIV) and related correlation techniques have been widely employed to visualize the velocity vector fields of either air or fuel phases for this purpose (Yamakawa et al. 2001; Hentschel et al. 1999; Krüger and Grünefeld 2000a; 2000b; 2000c; Kozma and Farrell 1997; Rhim and Farrell 2000; Lee et al. 2002). While velocity vector fields from the separate phases are useful, it is helpful to visualize the two phases simultaneously because of cycle-to-cycle variations of the fuel spray. Rottenkolber et al. $(1998,1999)$ proposed a method to discriminate between phases on a single double-exposure image, but this technique can assign phases incorrectly. Grünefeld et al. (2000) developed a technique based on a gaseous tracer (NO) in the air phase, but the setup is complicated for measurements performed at low temperatures. Khalitov and Longmire (2002) described a phase discrimination algorithm and a combination of particletracking and PIV to investigate two-phase flows (in their case, solid-gas). Towers et al. (1999) used a two-color approach whereby Mie scattering at the original, green, laser wavelength marked one phase, and fluorescence from doped particles identified the other phase. The same strategy was used by Lee and Nishida in investigations of DI gasoline sprays (2003). Both investigations have in common that only one double-pulse illumination is performed. In the case of the automotive fuel sprays with a large dynamic range of velocities this can limit the accuracy of the measurement in either phase since a compromise in the time difference of the two laser pulses has to be made. Thus, a new method was developed for the simultaneous measurement of air and fuel velocities in fuel sprays. Not only can it discriminate between phases, but it allows for the velocity resolution to be optimized based on the magnitude of the velocities probed in either phase.

\section{2}

\section{Experimental setup}

All measurements were performed using a pressure-swirl GDI spray $\left(60^{\circ}\right.$ cone angle), which was injected into a static test cell (1000 $\mathrm{cm}^{3}$ test volume) at room temperature and ambient pressure. The fuel was isooctane pressurized to $8.5 \mathrm{MPa}$ (injection duration $3 \mathrm{~ms}$ ) for the results presented here using a piston-type accumulator. The cell 


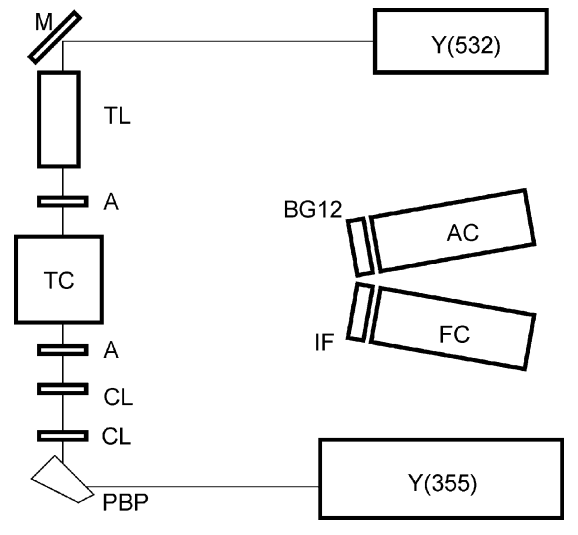

Fig. 1. Experimental setup: $Y(532)$, fuel visualization laser; $M$, dielectric mirror for $532 \mathrm{~nm}$; $T L$, telescopic lens system; $A$, aperture; $T C$, test cell; $B G 12$, bandpass filter; $I F$, interference filter; $A C$, air camera; $F C$, fuel camera; $C L$, cylindrical lens; $P B P$, PellinBroca prism; $Y(355)$, air visualization laser

was optically accessible on five sides through $75 \mathrm{~mm}$ diameter quartz windows. After each measurement set (five images per timing) a vacuum system was used to purge the cell.

A schematic of the experimental setup is given in Fig. 1. Mie scattering images were recorded from the spray, while air motion was tracked using fluorescent tracer particles seeded into the cell. The fuel phase measurement system consisted of a frequency-doubled double-pulse Nd:YAG laser (New Wave Mini-Lase, $30 \mathrm{~mJ} /$ pulse), sheet forming optics that focused a vertical light sheet through the center of the spray $(\approx 1$-mm sheet thickness, matched to the desired spatial resolution for the vector fields), and a doubleframe nonintensified CCD camera (LaVision Flowmaster3, $1280 \times 1024$ pixels per frame). A separation time of $8 \mu \mathrm{s}$ between laser pulses was chosen based on the desired resolution of the velocity vectors $(\approx 1 \mathrm{~mm})$ and the expected velocity range $(\approx 100 \mathrm{~m} / \mathrm{s})$. An interference filter was placed in front of the fuel-designated camera to filter out any interference from the air-phase signal.

The test cell was equipped with a droplet seeding system (Laskin-type nozzle), which introduced small seeding particles of ethylene glycol doped with Lambdachrome Stilbene 3 (428-nm peak fluorescence, 415-439-nm range, $15 \%$ fluorescence efficiency) into the test chamber prior to the experiment. PIV measurements of these tracer droplets taken before fuel injection confirm that the air in the cell was quiescent. The dye was excited with a frequency-tripled double-pulse Nd:YAG laser (Continuum Surelite $355 \mathrm{~nm}, 100-\mathrm{mJ} /$ pulse; the double-pulse operation with this laser was achieved with a second Marx bank to fire the Q-switch twice during one flashlamp event), and the resulting fluorescence was recorded on a second nonintensified CCD camera, identical to the one used for recording Mie scattering images. A BG-12 band-pass filter (407 nm, FWHM $104 \mathrm{~nm}$ ) was used to separate the LIF signal from Mie scattering from the spray occurring at $355 \mathrm{~nm}$ and also to suppress Mie scattering from the $532 \mathrm{~nm}$ lasers. A delay time of $80 \mu$ s between pulses was chosen based on the magnitude of air velocities and desired resolution. The laser pulses for the fuel measurement were triggered inside the timing pulses for the air phase as

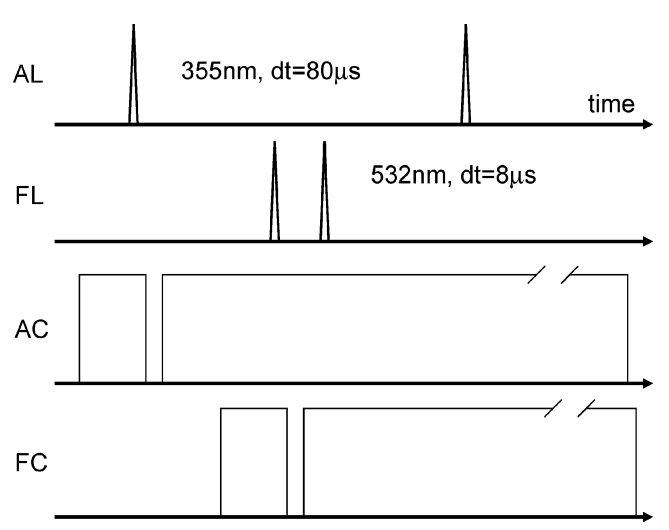

Fig. 2. Timing diagram: $A L$, air laser; $F L$, fuel laser; $A C$, air camera; $F C$, fuel camera. Camera gate width of first exposure is $10 \mu \mathrm{s}$; second is $125 \mathrm{~ms}$. Timing of the laser pulses is chosen such that the first pulse is fired during the first camera gate and the second pulse is fired during the second camera gate

illustrated in Fig. 2 for a simultaneous measurement of the two phases. The two laser systems, two cameras, and injector were synchronized using two programmable timing units that were part of the camera systems.

The air-phase droplets are smaller, and their number density is substantially less than that of the fuel droplets. Thus, the fuel images did not show measurable signals from the air phase. Furthermore, the air-phase droplets will have been displaced only a small amount in the time between the spray phase pulses, and any vector processed with this inadequately short pulse separation for the air velocity would result in near-zero displacement. Given the intense Mie scattering signal from the fuel, a reduced level of signal from the droplet phase is, however, present in the images for the air phase. Correlation for these droplet images is poor since the pulse separation $(80 \mu$ s for the air phase) is too long to ensure that both droplet images from the fuel are within the correlation window. Attempts to analyze the fuel images with particle tracking algorithms did not result in satisfactory results. Particle tracking is most appropriate where individual particle images are distinctly resolved from their neighbors by distances that are at least as large as the particle displacements. This condition is far from satisfied in the dense GDI spray being examined here.

Image processing involved the correction of images from each phase for distortion because of the slight inclination of the optical axis with respect to the normal to the laser light sheets (Fig. 1) and also matching of the spatial coordinates. The exact overlap of images from the two cameras is not critical for the success of the technique since velocities of a given phase are determined using a cross-correlation algorithm that is applied to the doubleframe image recorded with a single camera. The images from each camera were remapped to exactly coincide with each other as if both cameras were viewing the field of view from exactly the same perspective. This was achieved by calibrating each camera to a target grid of crosses positioned at the plane of the light sheet in the cell. The crosses are measured to within $0.05-0.1$ pixels, being based on the same cross-correlation algorithm as the measured particle displacements, and these positions combined with the 
precisely known grid spacing to generate a polynomial mapping function using a least squares fit to the data. The final registration of the remapped images is better than the uncertainty of the individually measured crosses and so therefore conservatively better than $0.1 \times 1 \mathrm{~mm} / 32=3 \mu \mathrm{m}$. The double-frame images from both cameras were processed separately to determine the corresponding vector fields. A sliding background (minimum) with a 5-pixel filter size was subtracted from the raw images to increase accuracy and reliability of the velocity results. A multipass cross-correlation strategy with a final interrogation window size of $32 \times 32$ pixels and $50 \%$ overlap was chosen to evaluate the velocities. Images were also post-processed restricting the allowed velocity range to remove erroneous vectors and to mask noise from outside the measurement area.

\section{3}

\section{Results and discussion}

The resolution of the velocity measurements is determined by the size of the interrogation region chosen. A resolution of $1 \mathrm{~mm}$ was desirable for both air and fuel since it provides enough detail for study and comparison against modeling results without exceeding the limitations of the equipment and technique. Since the velocities of the entrained air were an order of magnitude lower than those of the injected fuel, the delay between laser pulses needed to be adjusted for each phase so the 1-mm resolution could be attained in both phases. These delay times were chosen by limiting displacement of either phase to approximately one quarter of the interrogation region. The results presented below use a delay time of $8 \mu$ s for fuel and $80 \mu$ s for air, based on estimated maximum velocities of fuel (50$100 \mathrm{~m} / \mathrm{s})$ and air $(5-10 \mathrm{~m} / \mathrm{s})$. Since there is a large range of fuel velocities in the spray, the fuel delay may also be optimized in this manner to study specific regions in the spray with increased accuracy.

An example of the velocity fields that were obtained simultaneously from both phases is shown in Fig. 3. The air entrainment results (shown at $8.5 \mathrm{MPa}$ injection pressure, $0.54 \mathrm{~ms}$ after start of injection) adequately capture the air movement as it is pushed outward by the prespray and recirculated at the edge of the main spray. The fuel velocity data illustrate the predominantly downward direction of the fuel droplet flow. The most significant problem associated with measuring the velocity of the fuel phase using PIV is due to the dense nature of the GDI spray. For PIV ideally one requires clearly resolved images of the droplets whose velocities are being measured. In the fuel spray the number density of droplets is such that images of droplets overlap to some degree, creating partial speckle at the image plane. The speckle component of the image will decorrelate between laser pulses especially if there is out-of-plane motion (Adrian 1984). The subtraction of a sliding minimum improves the correlation of the fuel Mie scattering images. We also tried to eliminate speckle by doping the fuel with $1 \% 3$-pentanone and excited with a frequency-quadrupled Nd:YAG laser $(3 \mathrm{~mJ} /$ pulse). Fluorescence signals were too weak to be detected with the nonintensified camera, and an intensified camera was not desirable since it would further blur results). Thus,

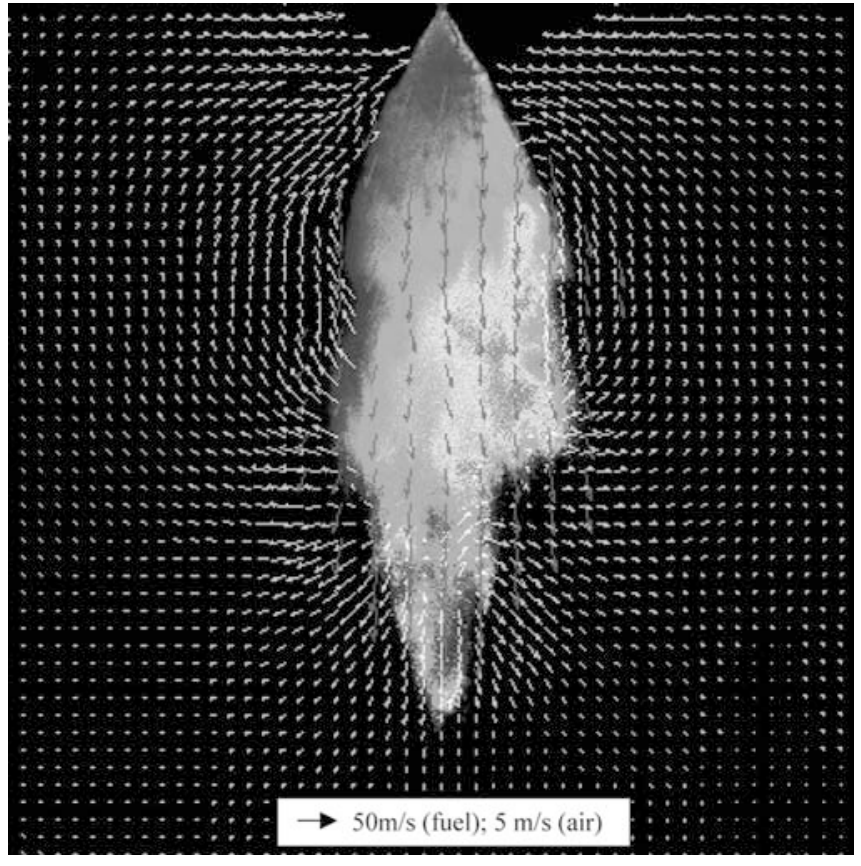

Fig. 3. Simultaneously measured air velocities (white arrows) and fuel velocities (blue vectors) in a single spray event (8.5 MPa injection pressure, $0.54 \mathrm{~ms}$ after start of injection). Fuel velocities are 10 times larger than air velocities for a given vector length. Also note that for clarity only every second fuel velocity vector in each direction is displayed. The background image is Mie scattering from the liquid fuel droplets Note that Fig. appears in grayscale format

Mie scattering was used to visualize the fuel spray in all experiments.

\section{Summary and conclusions}

A new technique was demonstrated for the simultaneous determination of entrained air velocities and fuel velocities in a dense spray. This method allows for unambiguous phase separation and optimal adaptation to best velocity resolution through the adjustment of laser delay times. The ability to achieve simultaneous velocity measurements with $1-\mathrm{mm}$ resolution will assist efforts to model atomization and mixing in dense sprays.

\section{References}

Adrian RJ (1984) Scattering particle characteristics and their effect on pulsed laser measurement of fluid flow: speckle velocimetry versus particle image velocimetry. Appl Opt 23:1690-1691

Grünefeld G, Finke H, Bartelheimer J, Krüger S (2000) Probing the velocity fields of gas and liquid phase simultaneously in a twophase flow. Exp Fluids 29:322-330

Hentschel W, Homberg A, Ohmstede G, Müller T, Grünefeld G (1999) Investigation of spray formation of DI gasoline hollow-cone injectors inside a pressure chamber and glass ring engine by multiple optical techniques. SAE paper 1999-01-3660

Khalitov DA, Longmire EK (2002) Simultaneous two-phase PIV by two-parameter phase discrimination. Exp Fluids 32:252-268

Kozma J, Farrell PV (1997) Air entrainment in a high pressure diesel spray. SAE paper 971620

Krüger S, Grünefeld G (2000a) Droplet velocity and acceleration measurements in dense sprays by laser flow tagging. Appl Phys B $71: 611-615$ 
Krüger S, Grünefeld G (2000b) Gas-phase velocity field measurements in dense sprays by laser-based flow tagging. Appl Phys B 70:463466

Krüger S, Grünefeld G (2000c) Planar velocity measurements of the gas and liquid phase in dense sprays by flow tagging. Proc 10th international symposium on applications of laser techniques to fluid mechanics, Lisbon, $10-13$ July 2000

Lee J, Nishida K (2003) Simultaneous flow field measurement of DI gasoline spray and entrained ambient air by LIF-PIV technique. SAE paper 2003-01-1115

Lee J, Yamakawa M, Isshiki S, Nishida K (2002) An analysis of droplets and ambient air interaction in a DI gasoline spray using LIF-PIV technique. SAE paper 2002-01-0743

Miles P, Megerle M, Nagel Z, Reitz R, Sick V (2002) Turbulence production and Reynolds stress modeling in swirl-supported, direct-injection diesel engines. Proc Combust Inst, vol 29, 719-726
Rhim DR, Farrell PV (2000) Characteristics of air flow surrounding non-evaporating transient diesel sprays. SAE paper 2000-01-2789

Rottenkolber G, Dullenkopf K, Wittig S (1998) Two-phase PIV measurements inside the intake port of an IC-engine. Proceedings of VSJ-SPIE98, Yokohama, 6-9 December 1998

Rottenkolber G, Gindele J, Raposo J, Dullenkopf K, Hentschel W, Wittig S, Spicher U, Merzkirch W (1999) Spray analysis of a gasoline direct injector by means of simultaneous two-phase PIV. PIV'99, Santa Barbara, 16-18 September 1999

Towers DP, Towers CE, Buckberry CH, Reeves M (1999) A colour PIV system employing fluorescent particles for two phase flow measurements. Measurement Sci Technol 10:824-830

Yamakawa M, Isshiki S, Lee J, Nishida K, (2001) 3-D PIV analysis of structural behavior of DI gasoline spray. SAE paper 2001-01-3669

Zhao F, Harrington DL, Lai MC (2002) Automotive gasoline directinjection engines. SAE, Warrendale, PA 